\title{
A Real-World Assessment of Outcomes, Health Resource Utilization, and Costs Associated with Cerebral Edema in US Patients with Large Hemispheric Infarction
}

\author{
Nicole Tsao ${ }^{1} \mathbb{D} \cdot$ Qiang Hou $^{1} \mathbb{D} \cdot$ Shih-Yin Chen ${ }^{1} \mathbb{D} \cdot$ Steven R. Messe ${ }^{2} \mathbb{D}$
}

Accepted: 25 July 2021 / Published online: 22 August 2021

(c) The Author(s) 2021

\begin{abstract}
Background Patients with large hemispheric infarction (LHI) are at risk of cerebral edema (CED). This study analyzed health resource use, costs, and outcomes during and after acute hospitalization for LHI in US patients with and without CED. Methods Using IBM ${ }^{\circledR}$ MarketScan ${ }^{\circledR}$ Commercial, Medicaid, and Medicare databases, patients with incident hospitalization for LHI (International Classification of Diseases, Tenth Revision, Clinical Modification diagnosis codes of I63.03x, I63.13x, I63.23x, I63.31x, I63.41x, I63.51x) from 31 March 2016 through 31 December 2018 were identified and further categorized by the presence or absence of CED based on related diagnosis codes or a procedure code of craniectomy. Health resource use, costs, and outcomes were compared in patients with and without CED during hospitalization and after discharge.

Results Of 7336 Commercial, 1946 Medicaid, and 5015 Medicare patients with LHI, 7.8\%, 6.9\%, and 4.3\% had CED, respectively. After adjusting for age, sex, and baseline comorbidities, differences (95\% confidence intervals) in mean total costs of the index hospitalization in patients with CED versus without CED were $\$ 65,572$ (\$56,506-\$76,335), $\$ 44,395$ (\$26,442$\$ 63,495)$, and $\$ 31,417(\$ 18,982-\$ 48,543)$ in the Commercial, Medicaid, and Medicare groups, respectively. Similarly, the adjusted differences (95\% confidence intervals) in mean lengths of stay between patients with CED and without CED were 11.75 (10.17-13.48), 10.84 (7.59-14.17), and 3.69 (2.40-5.19) days, respectively. Mortality during index hospitalization was 10-20 times greater in patients with CED versus without CED $(p<0.0001)$. In those patients who survived and had at least 30-days of follow-up after discharge, CED was also associated with higher post-discharge resource utilization and costs in the commercially insured population who were younger than Medicare patients, and had fewer comorbidities than Medicare and Medicaid patients. This indicates the effect of CED after discharge was particularly burdensome for younger individuals. Conclusions In this large cohort study, inpatient mortality, health resource utilization and costs were consistently higher in patients with LHI who developed CED than in those without CED. These findings underscore the need for greater awareness of CED among policymakers and healthcare practitioners.
\end{abstract}

\section{Introduction}

Large hemispheric infarction (LHI), a severe stroke affecting the total or nearly total middle cerebral artery territory, with or without involvement of other arteries, accounts for up

Nicole Tsao was an employee of Biogen, Cambridge, MA, USA, at the time this study was conducted and is currently an employee of Sanofi Genzyme.

Shih-Yin Chen

sharon.chen@biogen.com

Biogen, Cambridge, MA, USA

2 Department of Neurology, Hospital of the University of Pennsylvania, Philadelphia, PA, USA to $10 \%$ of ischemic strokes [1-3]. Patients with an LHI are at risk for adverse outcomes, including urinary tract infection, aspiration pneumonia, pulmonary embolism, deep vein thrombosis, and cerebral edema (CED), which can lead to additional brain injury $[4,5]$.

This life-threatening edema may affect approximately half of those with LHI [6]. CED begins shortly after the onset of LHI and peaks at between 24 and $96 \mathrm{~h}$. When blood flow is disrupted, cell membrane ionic transport is impaired, leading to an influx of water into astrocytes, neurons, and other cells in the central nervous system. This causes cellular swelling and death, which is then followed by an influx of fluid and solutes into extracellular space in the brain, causing edema [6]. Thus, typically, within 2-3 days of the onset of LHI, patients who develop CED experience increased intracranial 


\section{Key Points for Decision Makers}

In patients with large hemispheric infarction, those who develop cerebral edema (CED) have greater utilization of health resources, higher adjusted costs, and longer adjusted lengths of stay during the index hospitalization than those without CED.

Patients who develop CED have 10-20 times greater mortality during the index hospitalization.

The impact of CED on costs and utilization of health resources after hospital discharge is particularly burdensome for younger patients.

pressure and brain herniation, which can lead to brain tissue compression, midline shift, and death [7-9]. Patients with LHI and severe malignant CED have a mortality rate of approximately $50-80 \%[9,10]$.

Few studies have examined the costs of CED in LHI except in the context of surgical decompression [11, 12]. Hence, more data are needed from real-world settings to document the health resource utilization and direct cost burden of LHI with accompanying CED. This study aimed to estimate the effect of CED on outcomes, health resource use, and healthcare-associated costs during acute hospitalization and after discharge in a cohort of US patients with LHI. It is hoped that these findings will aid in understanding the current healthcare resources needed to manage CED, quantifying the unmet needs in patients with CED, and supporting the evaluation of interventions or treatment strategies for CED.

\section{Methods}

\subsection{Study Design}

This was an observational, retrospective cohort study using data from the IBM ${ }^{\circledR}$ MarketScan ${ }^{\circledR}$ Commercial, Medicaid, and Medicare Supplemental Databases (IBM Corporation, Armonk, NY, USA) [13]. The IBM ${ }^{\circledR}$ MarketScan ${ }^{\circledR}$ Commercial Database contains data from several million individuals who are covered by employer-sponsored private health insurance. The Medicaid Database contains data for more than 22 million Medicaid enrollees, and the Medicare Supplemental Database contains data for individuals with Medicare Supplemental Insurance paid by employers, and includes the
Medicare-covered portion of payment, employer-paid portion, and out-of-pocket patient expenses. Data in all three databases include service-level claims for inpatient and outpatient services and outpatient prescription drugs and are fully anonymized to be compliant with the Health Insurance Portability and Accountability Act of 1996.

Data from 1 October 2015 to 30 September 2019 were used in the analysis. Patients were included if they had an incident hospitalization episode for LHI between 31 March 2016 and 31 December 2018. Because no International Classification of Diseases, Tenth Revision, Clinical Modification (ICD-10-CM) diagnosis code for LHI exists, codes for cerebral infarction of the middle cerebral artery or carotid artery (I63.03x, I63.13x, I63.23x, I63.31x, I63.41x, I63.51x in any position) were used as surrogates to identify LHI, as no other validated approaches have been described. Patients had to be enrolled continuously in medical and prescription drug plans during the 180 days (washout period) before the index date, defined as the first day of hospitalization for ischemic stroke. Individuals had to be aged 18-85 years at the index date and were excluded if they had any codes for prior inpatient or outpatient claims for ischemic stroke or ICD-10-CM codes for diagnosis of hemorrhagic stroke, cerebellar stroke, sepsis, head injury, brain tumors, or select cerebrovascular diseases during the index hospitalization (exclusion codes are shown in Table $\mathrm{S} 1$ in the electronic supplementary material $[\mathrm{ESM}])$. Medicaid patients with capitation plans or dual eligibility were excluded from the analysis due to incomplete capture of cost data.

Patients included in the analysis were categorized by the presence or absence of CED, which was identified by ICD-10 codes G93.5 (compression of brain) or G93.6 (cerebral edema), or having undergone a craniectomy procedure (based on ICD-10-CM procedure system codes or Healthcare Common Procedure Coding System/Current Procedural Terminology codes as listed in Tables S2 and S3 in the ESM). Patients were followed until 30 September 2019 or disenrollment from the medical and drug plan (defined as either the last day of the last month of enrollment, or the date of the last claim within 30 days after the end of enrollment). All post-index hospitalization costs and health resource utilization were accounted for during follow-up until and including those that were incurred during the month of disenrollment, where non-informative censoring was assumed.

\subsection{Outcome Variables}

We described the clinical management of these patients during the index hospitalization, including tissue plasminogen activator administration, craniectomy/craniotomy, thrombectomy, tracheostomy or endotracheal intubation, brain imaging (computed tomography scan or magnetic resonance imaging), and in-hospital outcomes such as 
intensive care unit (ICU) admission, total costs, length of stay of index hospitalization, and mortality. Post-discharge outcomes included frequency of use of emergency department visits, inpatient stays, outpatient visits, long-term care facility stays, skilled nursing facility stays, prescriptions, and durable medical equipment use, as well as total healthcare costs over the follow-up period. Due to various length of follow-up, post-discharge utilization and total healthcare costs were annualized.

\subsection{Statistical Analysis}

Data were reported by insurance type (i.e., Commercial, Medicaid or Medicare status). Baseline characteristics included age, sex and Charlson Comorbidity Index (CCI). Clinical management and outcome variables were described with summary statistics and compared in patients with and without CED. Comparisons of continuous variables were analyzed using Wilcoxon rank-sum tests, and categorical variables were analyzed using Chi-square tests. Length of index hospital stay and total costs of the index hospitalization in patients with and without CED were analyzed using generalized linear models adjusted by age at index data, sex, and CCI with 500 random resamplings with replacement bootstrapping to determine 95\% confidence intervals (CIs). Annualized post-hospitalization total costs until the end of follow-up were analyzed using generalized linear models adjusted for baseline characteristics with 500 random resamplings with replacement bootstrapping to determine $95 \%$ CIs. Costs were reported at their valuation in the time they were collected (2016-2019) without adjustment. Incidence rate ratios (IRRs) for health resource utilization were determined using Poisson modeling and were adjusted for baseline characteristics. Multivariable logistic regression analysis was used to determine odds ratios [ORs] (95\% CIs) for inpatient mortality during the index hospitalization. These analyses were performed among those with complete data on the dependent and independent variables. All analyses were performed using the SAS 9.4 software (SAS Institute, Cary, NC, USA).

\section{Results}

\subsection{Cohort Characteristics}

A total of 101,194 patients in the MarketScan databases were identified as having been hospitalized for ischemic stroke between 31 March 2016 and 31 December 2018, and 14,297 with LHI who met all eligibility criteria, were included in the analysis (Fig. 1). The cohort categorized by insurance group included 7336 Commercial insurance patients, 1946 Medicaid patients, and 5015 Medicare patients. Of these,
572 (7.8\%), 135 (6.9\%), and 218 (4.3\%), respectively, had LHI with CED or craniectomy. Of the possible LHI patients, mean (standard deviation [SD]) age was 76.13 (5.88) years and mean (SD) CCI was 1.17 (0.91) in the Medicare group, reflecting the older population of Medicare beneficiaries. Although the mean (SD) age was similar between the Commercial and Medicaid groups [55.01 (8.67) and 55.51 (10.33), respectively], the Medicaid group had a higher mean CCI [1.3 (1.0), compared with $0.79(0.89)$ in the Commercial group], representing a more disabled population. Among Commercial and Medicaid patients, those with CED were significantly younger than those without CED $(p<0.0001)$ (Table 1). Medicaid patients without CED had slightly more comorbidities based on a higher CCI than those with CED $(p=0.0328)$ (Table 1). Across all three insurance groups, no difference was seen in the ratio of males to females between those with and those without CED (Table 1).

\subsection{Index Hospitalization Clinical Management}

During the index hospitalization for LHI, development of CED was associated with greater health resource use. Across all insurance groups, compared with patients without CED, those with CED had significantly higher rates of tissue plasminogen activator use ( $p<0.0001$, all insurance groups), thrombectomy procedures $(p<0.0001$, all insurance groups), tracheostomies or endotracheal intubations $(p<0.0001)$, and imaging procedures (Commercial insurance, $p<0.0001$; Medicaid, $p=0.0187$; Medicare, $p=0.0011$ ) (Table 1). Of the CED cohort, 16.3\%, 10.4\%, and $3.2 \%$ received craniectomy in the Commercial, Medicaid, and Medicare groups, respectively.

In all insurance groups, significantly smaller proportions of patients with CED were discharged to their homes $(p<0.001)$. Significantly higher proportions of commercially insured patients with CED were discharged to skilled nursing facilities or hospices, or died $(p<0.001)$, whereas significantly higher proportions of Medicaid patients with CED were discharged to skilled nursing facilities $(p=0.003)$ or died $(p<0.001)$, and significantly higher proportions of Medicare patients with CED were discharged to hospice or $\operatorname{died}(p<0.001)$.

\subsection{Index Hospitalization Outcomes}

The development of CED resulted in poorer clinical outcomes. In all three insurance groups, higher proportions of patients with CED were admitted to the ICU (76.1-89.6\% across insurance groups) than patients without CED (approximately 50\%) (Table 2). After adjusting for age at index date, sex, and CCI, patients with CED in the Commercial and Medicare groups were more than four (OR [95\% CI] 4.12 [3.28-5.12]; $p<0.0001)$ and three times (OR 3.24 


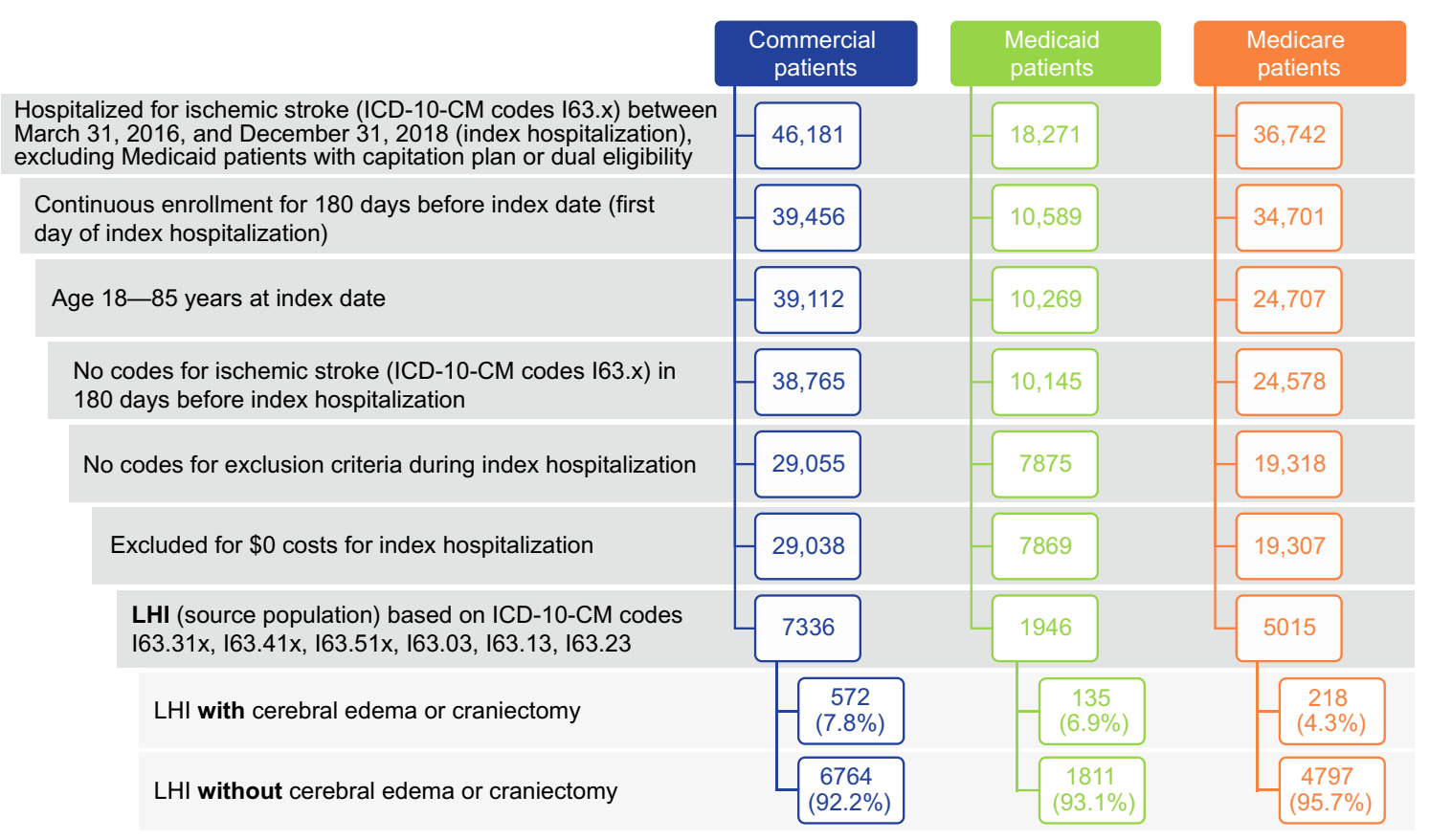

Fig. 1 Cohort attrition. LHI large hemispheric infarction, ICD-10-CM International Classification of Diseases, Tenth Revision, Clinical Modification

[2.36-4.45]; $p<0.0001)$ more likely to be admitted to the ICU, respectively, than those without CED, whereas in the Medicaid group, patients with CED were over 11 times more likely to be admitted (OR [95\% CI] 11.14 (6.33-19.60); $p<0.0001)$ than those without CED. Median length of hospital stay was longer in patients with CED (8-14 days) than in those without CED (4-6 days; unadjusted $p<0.0001$ ). After adjustment for baseline characteristics, hospital length of stay was significantly longer in patients with CED than those without CED in all insurance groups; the least square mean difference $(95 \% \mathrm{CI})$ between those with CED and those without CED was 11.75 (10.17-13.48) days in the Commercial group, 10.84 (7.59-14.17) days in the Medicaid group, and 3.69 (2.40-5.19) days in the Medicare group (Table 2).

Development of CED increased the likelihood of death following LHI. Higher proportions of patients with CED died (15.0-21.1\%) during the index hospitalization than those without CED (1.0-2.6\%) (Table 1). After adjustment for baseline characteristics, in the Commercial group, those with CED were almost 20 times more likely to die during the index hospitalization than those without CED (OR [95\% CI] 19.70 [13.97, 27.77]; $p<0.0001$ ) (Table 2). Similarly, in the Medicaid and Medicare groups, patients with CED were approximately 10 (OR [95\% CI] 10.30 [7.08-15.00]; $p<0.0001$ ) and 16 times (OR [95\% CI] 15.99 [8.83-28.97]; $p<0.0001)$ more likely to die during the index hospitalization, respectively (Table 2) than those without CED.

\subsection{Index Hospitalization Costs}

CED development increased the costs of index hospitalization for LHI. Crude total mean costs of index hospitalization in patients with CED were US\$107,608, US\$75,912, and US\$66,348 in the Commercial, Medicaid, and Medicare groups, respectively, and in patients without CED were US $\$ 39,799$, US $\$ 32,904$, and US $\$ 34,062$, respectively. After adjustment for baseline characteristics, total costs of hospitalization were significantly higher in patients with CED in all insurance groups versus patients without CED: the least square mean difference $(95 \% \mathrm{CI})$ was US $\$ 65,572$ (US\$56,506-US\$76,335) in the Commercial group, US\$44,395 (US\$26,442-US\$63,495) in the Medicaid group, and US\$31,417 (US\$18,982-US\$48,543) in the Medicare group (Table 2).

\subsection{Post-Index Hospitalization Health Resource Utilization}

Post-index hospitalization data were available for 13,187 patients, including 6904 Commercial, 1800 Medicaid, and 4483 Medicare patients (Table 3). Development of CED resulted in greater health resource utilization for commercially insured patients. In the Commercial group, compared with patients without CED, those with CED had significantly higher rates of all health care resources, including emergency department visits (IRR 1.5127; $p<0.0001$ ), inpatient readmission (IRR 1.8362; $p<0.0001$ ), outpatient 
Table 1 Patient demographics and characteristics, clinical management, and index discharge status distribution

\begin{tabular}{|c|c|c|c|c|c|c|c|c|c|}
\hline & \multicolumn{3}{|l|}{ Commercial } & \multicolumn{3}{|l|}{ Medicaid } & \multicolumn{3}{|l|}{ Medicare } \\
\hline & $\begin{array}{l}\text { CED } \\
{[n=572]}\end{array}$ & $\begin{array}{l}\text { Non-CED } \\
{[n=6764]}\end{array}$ & $p$ value & $\begin{array}{l}\text { CED } \\
{[n=135]}\end{array}$ & $\begin{array}{l}\text { Non-CED } \\
{[n=1811]}\end{array}$ & $p$ value & $\begin{array}{l}\text { CED } \\
{[n=218]}\end{array}$ & $\begin{array}{l}\text { Non-CED } \\
{[n=4797]}\end{array}$ & $p$ value \\
\hline $\begin{array}{l}\text { Mean }(\mathrm{SD}) \\
\text { age, years }\end{array}$ & $53.43(9.64)$ & $55.14(8.57)$ & $<0.0001$ & $50.06(11.85)$ & $55.91(10.1)$ & $<0.0001$ & $75.95(6.04)$ & $76.14(5.88)$ & 0.6397 \\
\hline \multicolumn{10}{|c|}{ Age category, years } \\
\hline $18-39$ & $54(9.4)$ & $419(6.2)$ & & $28(20.7)$ & $116(6.4)$ & & 0 & 0 & \\
\hline $40-64$ & $502(87.8)$ & $6133(90.7)$ & & $102(75.6)$ & $1500(82.8)$ & & $1(0.5)$ & $53(1.1)$ & \\
\hline$\geq 65$ & $16(2.8)$ & $212(3.1)$ & & $5(3.7)$ & $195(10.8)$ & & $217(99.5)$ & 4744 (98.9) & \\
\hline Sex & & & 0.397 & & & 0.477 & & & 0.0867 \\
\hline Male & $238(41.6)$ & $2938(43.4)$ & & $64(47.4)$ & $916(50.6)$ & & $120(55.0)$ & $2356(49.1)$ & \\
\hline Female & $334(58.4)$ & $3826(56.6)$ & & $71(52.6)$ & $895(49.4)$ & & $98(45.0)$ & $2441(50.9)$ & \\
\hline $\begin{array}{l}\text { Mean (SD) } \\
\text { CCI }\end{array}$ & $0.73(0.88)$ & $0.79(0.89)$ & 0.076 & $1.12(0.96)$ & $1.32(1)$ & 0.0328 & $1.10(0.97)$ & $1.17(0.91)$ & 0.1514 \\
\hline CCI category & & & 0.2797 & & & 0.0543 & & & 0.0742 \\
\hline 0 & $291(50.9)$ & 3154 (46.6) & & $44(32.6)$ & $446(24.6)$ & & $69(31.7)$ & $1217(25.4)$ & \\
\hline 1 & $171(29.9)$ & $2210(32.7)$ & & $42(31.1)$ & $615(34.0)$ & & $81(37.2)$ & $1937(40.4)$ & \\
\hline 2 & $82(14.3)$ & $1049(15.5)$ & & $38(28.1)$ & $479(26.4)$ & & $45(20.6)$ & $1236(25.8)$ & \\
\hline$\geq 3$ & $28(4.9)$ & $351(5.2)$ & & $11(8.1)$ & $271(15.0)$ & & $23(10.6)$ & $407(8.5)$ & \\
\hline \multicolumn{10}{|c|}{ Clinical management } \\
\hline $\begin{array}{l}\text { TPA (identi- } \\
\text { fied by } \\
\text { CPT, } \\
\text { HCPCS, } \\
\text { or PCS } \\
\text { codes) }\end{array}$ & $170(29.7)$ & $1298(19.2)$ & $<0.0001$ & 40 (29.6) & $240(13.3)$ & $<0.0001$ & $61(28.0)$ & $578(12.0)$ & $<0.0001$ \\
\hline $\begin{array}{l}\text { Craniec- } \\
\text { tomy/cra- } \\
\text { niotomy }\end{array}$ & $93(16.3)$ & 0 & $<0.0001$ & $14(10.4)$ & 0 & $<0.0001$ & $7(3.2)$ & 0 & $<0.0001$ \\
\hline $\begin{array}{l}\text { Thrombec- } \\
\text { tomy }\end{array}$ & $157(27.4)$ & $846(12.5)$ & $<0.0001$ & $28(20.7)$ & $125(6.9)$ & $<0.0001$ & $51(23.4)$ & $468(9.8)$ & $<0.0001$ \\
\hline $\begin{array}{l}\text { Tracheos- } \\
\text { tomy or } \\
\text { endotra- } \\
\text { cheal } \\
\text { intubation }\end{array}$ & $90(15.7)$ & $130(1.9)$ & $<0.0001$ & $31(23.0)$ & $69(3.8)$ & $<0.0001$ & $37(17.0)$ & $97(2.0)$ & $<0.0001$ \\
\hline $\begin{array}{l}\text { CT or MRI } \\
\text { of brain }\end{array}$ & $551(96.3)$ & $5973(88.3)$ & $<0.0001$ & $130(96.3)$ & $1633(90.2)$ & 0.0187 & $202(92.7)$ & $4056(84.6)$ & 0.0011 \\
\hline \multicolumn{10}{|c|}{ Index discharge status distribution } \\
\hline Home & $237(41.4)$ & $4754(70.3)$ & $<0.001$ & $24(17.8)$ & 900 (49.7) & $<0.001$ & $57(26.1)$ & $2388(49.8)$ & $<0.001$ \\
\hline $\begin{array}{l}\text { Skilled nurs- } \\
\text { ing facility }\end{array}$ & $64(11.2)$ & $293(4.3)$ & $<0.001$ & $32(23.7)$ & $260(14.4)$ & 0.003 & $26(11.9)$ & $610(12.7)$ & 0.732 \\
\hline Hospice & $16(2.8)$ & $29(0.4)$ & $<0.001$ & $2(1.5)$ & $23(1.3)$ & 0.833 & $23(10.6)$ & $103(2.1)$ & $<0.001$ \\
\hline Death & $86(15.0)$ & $65(1.0)$ & $<0.001$ & $26(19.3)$ & $29(1.6)$ & $<0.001$ & $46(21.1)$ & $124(2.6)$ & $<0.001$ \\
\hline $\begin{array}{l}\text { Other dis- } \\
\text { charge }\end{array}$ & $122(21.3)$ & $1116(16.5)$ & 0.003 & $47(34.8)$ & $589(32.5)$ & 0.584 & $53(24.3)$ & $1420(29.6)$ & 0.094 \\
\hline $\begin{array}{l}\text { Unknown } \\
\text { discharge }\end{array}$ & $47(8.2)$ & $507(7.5)$ & 0.531 & $10(7.4)$ & $4(0.2)$ & 0.001 & $13(6.0)$ & $146(3.0)$ & 0.016 \\
\hline
\end{tabular}

Data are expressed as $n(\%)$ unless otherwise specified

CCI Charlson Comorbidity Index, CED cerebral edema, CPT Current Procedural Terminology, CT computed tomography, HCPCS Healthcare Common Procedure Coding System, MRI magnetic resonance imaging, PCS procedure coding system, $S D$ standard deviation, $T P A$ tissue plasminogen activator 
Table 2 Index hospitalization outcomes and costs

\begin{tabular}{|c|c|c|c|c|}
\hline & CED & Non-CED & Unadjusted $p$ value & $\begin{array}{l}\text { OR }(95 \% \mathrm{CI})^{\mathrm{a}} / \mathrm{LS} \text { mean } \\
\text { difference }(95 \% \mathrm{CI})^{\mathrm{b}}\end{array}$ \\
\hline Commercial & $(n=572)$ & $(n=6764)$ & & \\
\hline ICU admission $[n(\%)]$ & $478(83.6)$ & $3720(55.0)$ & $<0.0001$ & $\begin{array}{l}4.12(3.28-5.17) \\
p<0.0001\end{array}$ \\
\hline Median (SD) length of stay, days & $12(9.55)$ & $4(5.11)$ & $<0.0001$ & $11.75(10.17-13.48)^{\mathrm{c}}$ \\
\hline Inpatient mortality $[n(\%)]$ & $86(15.0)$ & $65(1.0)$ & $<0.001$ & $\begin{array}{l}19.70(13.97-27.77) \\
p<0.0001\end{array}$ \\
\hline Mean (SD) total costs, US\$ & $107,608(116,755)$ & $39,799(50,681)$ & $<0.0001$ & $65,572(56,506-76,335)^{\mathrm{c}}$ \\
\hline Medicaid & $(n=135)$ & $(n=1811)$ & & \\
\hline ICU admission $[n(\%)]$ & $121(89.6)$ & $825(45.6)$ & $<0.0001$ & $\begin{array}{l}11.14(6.33-19.60) \\
p<0.0001\end{array}$ \\
\hline Median (SD) length of stay, days & $14(49.67)$ & $6(13.43)$ & $<0.0001$ & $10.84(7.59-14.17)^{\mathrm{c}}$ \\
\hline Inpatient mortality $[n(\%)]$ & $26(19.3)$ & $29(1.6)$ & $<0.001$ & $\begin{array}{l}10.30(7.08-15.00) \\
p<0.0001\end{array}$ \\
\hline Mean (SD) total costs, US\$ & $75,912(110,655)$ & $32,904(45,697)$ & $<0.0001$ & $44,395(26,442-63,495)^{\mathrm{c}}$ \\
\hline Medicare & $(n=218)$ & $(n=4797)$ & & \\
\hline ICU admission $[n(\%)]$ & $166(76.1)$ & $2373(49.5)$ & $<0.0001$ & $\begin{array}{l}3.24(2.36-4.45) \\
p<0.0001\end{array}$ \\
\hline Median (SD) length of stay, days & $8(18.16)$ & $5(7.55)$ & $<0.0001$ & $3.69(2.40-5.19)$ \\
\hline Inpatient mortality $[n(\%)]$ & $46(21.1)$ & $124(2.6)$ & $<0.001$ & $\begin{array}{l}15.99(8.83-28.97) ; \\
p<0.0001\end{array}$ \\
\hline Mean (SD) total costs, US\$ & $66,348(106,331)$ & $34,062(59,561)$ & $<0.0001$ & $31,417(18,982-48,543)$ \\
\hline
\end{tabular}

$C E D$ cerebral edema, $C I$ confidence interval, $I C U$ intensive care unit, $L S$ least square, $O R$ odds ratio, $S D$ standard deviation

${ }^{\mathrm{a}} \mathrm{ORs}, 95 \% \mathrm{CIs}$, and $p$ values were based on a logistic regression model

${ }^{b}$ LS means (95\% CIs) from generalized linear models adjusted by age at index data, sex, and Charlson Comorbidity Index (capped by 3 ) and bootstrapped with 500 random resampling with replacement

${ }^{c}$ Outliers (two Commercial and seven Medicaid patients) were not included because their length of stay exceeded 10 times that of the pooled SDs

visits (IRR 1.2163; $p<0.0001$ ), prescription medication (IRR 1.2943; $p<0.0001$ ), use of durable medical equipment (IRR $4.3861 ; p<0.0001$ ), and skilled nursing facility stays (IRR 4.2561; $p=0.001$ ) (Table 3). In the Medicaid group, patients with CED had significantly higher rates of stay in long-term care facilities (IRR 2.586; $p<0.0001$ ) and skilled nursing facilities (IRR 2.389; $p<0.0001$ ) than patients without CED. In the Medicare group, patients with CED had significantly higher rates of prescription medication use (IRR $1.1733 ; p=0.0249$ ) and stays in skilled nursing facilities (IRR $1.8891 ; p=0.001$ ) than patients without CED.

The development of CED resulted in significantly greater costs for commercially insured and Medicare patients. The crude post-discharge annualized total healthcare cost per patient in patients with CED was US\$77,091, US\$71,640, and US\$63,047 in the Commercial, Medicaid, and Medicare groups, respectively, and $\$ 52,090, \$ 63,287$, and $\$ 49,813$, respectively, in patients without CED. After adjustment for age at index data groups, sex, and CCI, post-discharge annualized total costs per patient were significantly greater in patients with CED than those without CED in the Commercial insurance group (least square mean difference
[95\% CI] US $\$ 30,171$ [US\$18,678-US\$45,568]) and the Medicare group (least square mean difference [95\% CI] US $\$ 20,451$ [US\$4456-US\$38,588]), but not in the Medicaid group (least square mean difference [95\% CI] US $\$ 25,111$ [-US\$3,899 to US\$62,851]) (Fig. 2).

\section{Discussion}

In this real-world study of a large cohort of patients with LHI, we examined the impact of CED on outcomes, health resource utilization, and healthcare-associated costs during the index hospitalization and after discharge. While the findings are limited to the individuals covered by employersponsored private health insurance and Medicaid in several states, to our knowledge this is the largest study to date with data from a combined 125 million covered lives. The study demonstrated that patients with LHI and CED had greater utilization of health resources, higher adjusted costs, and longer adjusted lengths of stay during the index hospitalization than those without CED, across the three types of insurance enrollees examined in this study. These patients 
Table 3 Health resource utilization following index hospitalization discharge in patients who survived and remained enrolled for at least 30 days

\begin{tabular}{|c|c|c|c|c|}
\hline & CED & Non-CED & $\mathrm{IRR}^{\mathrm{a}}$ & $p$ value \\
\hline Commercial & $n=572$ & $n=6764$ & & \\
\hline Patients continuously enrolled post-index hospitalization $[n(\%)]$ & $453(79.2)$ & $6451(95.4)$ & & \\
\hline Mean (SD) follow-up, months & $11.0(10.59)$ & $15.73(11.13)$ & & \\
\hline \multicolumn{5}{|l|}{ Post-index hospitalization resource use, annualized frequencies (SD) } \\
\hline ED visits & $3.08(6)$ & $2.40(6.36)$ & 1.51 & $<0.0001$ \\
\hline Inpatient stay & $0.95(1.91)$ & $0.59(1.73)$ & 1.84 & $<0.0001$ \\
\hline Outpatient visits & $17.5(15.76)$ & $15.74(16.04)$ & 1.22 & $<0.0001$ \\
\hline Number of prescriptions & $40.68(44.83)$ & $34.41(30.75)$ & 1.29 & $<0.0001$ \\
\hline DME use & $3.3(7.77)$ & $1.02(4.49)$ & 4.39 & $<0.0001$ \\
\hline LTC facility stays & - & - & - & - \\
\hline SNF stays & $2.17(5.77)$ & $0.7(4.04)$ & 4.26 & $<0.0001$ \\
\hline Medicaid & $n=135$ & $n=1811$ & & \\
\hline Patients continuously enrolled post-index hospitalization $[n(\%)]$ & $103(76.3)$ & $1697(93.7)$ & & \\
\hline Follow-up, months (SD) & $9.68(9.57)$ & $14(9.51)$ & & \\
\hline \multicolumn{5}{|l|}{ Post-index hospitalization resource use, annualized frequencies (SD) } \\
\hline ED visits & $3.91(6.56)$ & $4.32(6.97)$ & 1.03 & 0.8372 \\
\hline Inpatient stay & $1.19(2.24)$ & $1.31(2.38)$ & 0.97 & 0.855 \\
\hline Outpatient visits & $12.53(10.96)$ & $14.36(12.37)$ & 0.92 & 0.2741 \\
\hline Number of prescriptions & $70.17(70.92)$ & $81.05(72.61)$ & 1.02 & 0.8544 \\
\hline DME use & $4.76(12.91)$ & $3.85(24.44)$ & 1.95 & 0.1757 \\
\hline LTC facility stays & $7.03(15.08)$ & $3.27(9.36)$ & 2.59 & $<0.0001$ \\
\hline SNF stays & $2.36(5.35)$ & $1.13(3.15)$ & 2.39 & $<0.0001$ \\
\hline Medicare & $n=218$ & $n=4797$ & & \\
\hline Patients continuously enrolled post-index hospitalization $[n(\%)]$ & $141(64.7)$ & $4342(90.5)$ & & \\
\hline Follow-up, months (SD) & $8.28(10.57)$ & $14(11.34)$ & & \\
\hline \multicolumn{5}{|l|}{ Post-index hospitalization resource use, annualized frequencies (SD) } \\
\hline ED visits & $4.5(8.91)$ & $4.36(9.43)$ & 1.22 & 0.2046 \\
\hline Inpatient stay & $0.66(1.66)$ & $0.57(1.51)$ & 1.26 & 0.1614 \\
\hline Outpatient visits & $14.08(12.82)$ & $15.60(15.87)$ & 1.11 & 0.144 \\
\hline Number of prescriptions & $38.15(40.5)$ & $40.14(35.68)$ & 1.17 & 0.0249 \\
\hline DME use & $2.19(6.25)$ & $1.76(6.01)$ & 1.36 & 0.2576 \\
\hline LTC facility stays & - & - & & \\
\hline SNF stays & $6.11(11.61)$ & $3.00(8.01)$ & 1.89 & 0.001 \\
\hline
\end{tabular}

$C E D$ cerebral edema, $D M E$ durable medical equipment, $E D$ emergency department, $I R R$ incidence rate ratio, $L T C$ long-term care, $S D$ standard deviation, $S N F$ skilled nursing facility

${ }^{a}$ Based on Poisson regression analysis adjusted for age, sex, and Charlson Comorbidity Index

also had 10-20 times greater mortality during the index hospitalization than patients without CED. Across the three insurance populations, CED consistently led to significant economic consequences to the healthcare system and worse clinical outcomes.

After discharge from index hospitalization, patients with CED incurred approximately US $\$ 20,000-$ US $\$ 30,000$ more in annual total healthcare-related costs compared with those without CED. Those with CED in the Commercial group had significantly greater health resource utilization of all resources than those without CED. Most health resource utilization and costs in the Medicaid and Medicare groups were not statistically significantly higher in patients with CED. Individuals insured under the Commercial plans were younger than those with Medicare insurance, and had fewer comorbidities than Medicare and Medicaid patients. This study suggests that CED occurring during hospitalization had a more profound impact in this relatively healthier cohort after they were discharged, at least partially reflecting the fact that older patients or those with more comorbidities do poorly after LHI regardless of CED. It is possible that in a frail and disabled population such as those in the Medicare or Medicaid groups, the most significant healthcare resource impact related to CED was at the index hospitalization, and, 


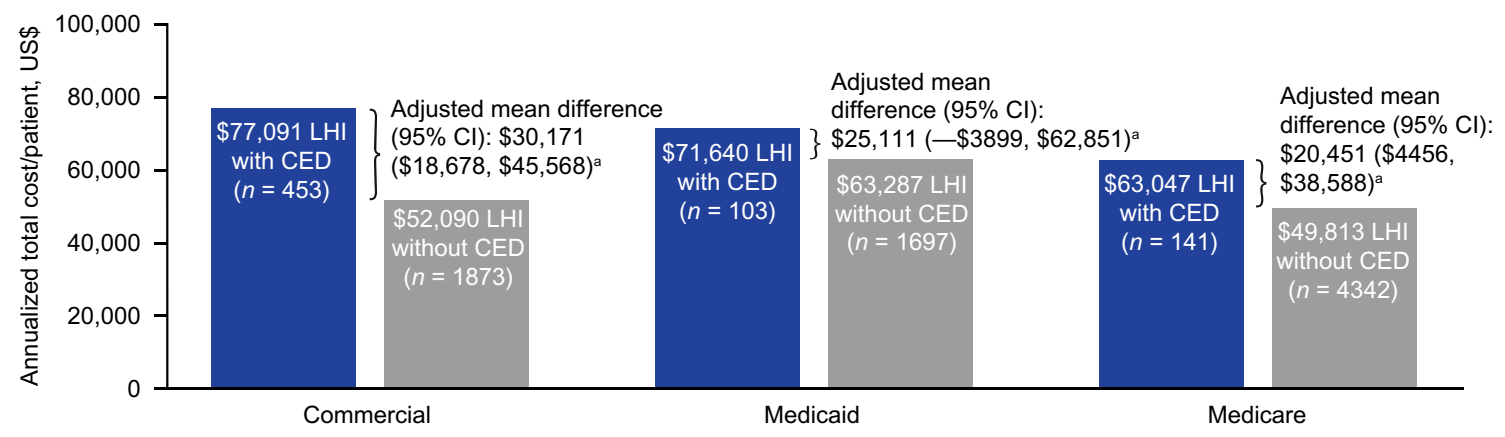

Fig. 2 Total annualized healthcare costs following index hospitalization discharge in patients who survived and remained enrolled for at least 30 days. ${ }^{a}$ Based on generalized linear models adjusted by age at index data groups, sex, and Charlson Comorbidity Index (capped by
3), and bootstrapped with 500 random resampling with replacement. CED cerebral edema, $C I$ confidence interval, $L H I$ large hemispheric infarction once they survived, the relative incremental utilization was not as large due to the underlying health status. Nevertheless, the sample sizes were relatively smaller in the Medicaid and Medicare groups, limiting our power to detect potentially meaningful differences, and future study should aim at confirming these findings with a larger sample.

In this study, across insurance groups the rate of inpatient mortality during the index hospitalization in patients with CED ranged from 15 to $21 \%$. In contrast, previous studies reported mortality rates of $70-78 \%$ after up to 8 days and a 1-month mortality rate of $59 \%$ in patients receiving nonsurgical treatment (including artificial hyperventilation, barbiturate coma, and hyperosmolar therapy) $[9,19,20]$. It is likely that the lower mortality rate we observe reflects the fact that decompressive hemicraniectomy has become standard of care for malignant middle cerebral artery infarction and has been shown to improve survival to the range of $25-30 \%$ [16, 20, 21]. Furthermore, given that we did not have any direct data on stroke severity or infarct volume, it is possible that we included patients with smaller, milder strokes who had some degree of brain edema, but not malignant infarction.

Approximately $6 \%$ of the LHI patients were classified as having developed CED in this study, which is substantially lower than the $50 \%$ previously reported [6]. Again, this may be due to potential misclassification of CED due to variation in ICD coding practices or the inclusion in the cohort of less severe patients with LHI who may not have been at a high risk of edema compared with clinically confirmed LHI cases.

Rapid tissue reperfusion is recommended for acute ischemic stroke to prevent subsequent CED [22]; however, this may not apply to LHI. Once CED develops after LHI, therapeutic options are limited. Nonsurgical management options are considered safe, but evidence of benefit from large prospective randomized studies is lacking [14]. In this study, use of acute stroke interventions including tissue plasminogen activator and thrombectomy, as well as invasive procedures including tracheostomy and endotracheal intubation, were significantly more common in patients who developed CED, reflecting that these patients likely had more severe strokes. Craniectomy and craniotomy, at least one of which was utilized in $3.2-16.3 \%$ of CED patients during index hospitalization in this study, are also associated with substantive morbidity, especially among patients aged $>60$ years $[2,3,15,16]$. Furthermore, a meta-analysis of surgical decompression studies showed high rates of morbidity despite decreased mortality in patients who undergo surgical treatment for edema [17]. Consistent with previous studies of LHI [18], patients who developed CED were younger than patients who did not develop CED in both the privately insured and Medicaid cohorts, and the incidence was higher in these cohorts relative to the older Medicare group. Given this increased risk of CED in the younger commercially insured population, and the more significant postdischarge impact of CED, this group likely has the greatest opportunity to benefit from novel therapies for CED.

\subsection{Limitations}

Although in a large cohort, this study has a number of limitations, including a lack of information on imaging and stroke severity as noted above. As a result, the diagnosis of LHI (source population) and development of CED could not be clinically confirmed or severity of edema ascertained. Instead, the identification of LHI should be considered with caution because while we relied on the most appropriate set of diagnosis codes, this approach has not been validated. Because only about $50 \%$ of patients with an infarct of the middle cerebral artery are thought to develop LHI [23], it is possible that some patients included in the LHI cohort did not actually have an LHI, which was evidenced by the lower proportion of patients with CED identified in this study than the literature. Therefore, the extent of the observed burden 
associated with CED may be somewhat overestimated. A future study to evaluate the validity of the algorithm through a manual chart review would be helpful to inform future research. Although multiple regression analysis was used to adjust covariates identified for the comparisons to reduce confounders, this method, unlike randomization, cannot remove unmeasured confounders. This remains a limitation of the study using the claims database analysis. Finally, our analysis was not able to account for costs that were incurred by individuals who had dual eligibility in Medicaid and Medicare, or those with capitation plans in Medicaid.

\section{Conclusion}

The results of this large, real-world study show that CED in patients with LHI was associated with greatly increased inpatient mortality, health resource utilization, and costs across the insurance populations examined, while the effect of CED after hospital discharge was particularly burdensome for younger populations. These findings suggest there should be a greater appreciation of CED among policymakers and healthcare practitioners.

Supplementary Information The online version contains supplementary material available at https://doi.org/10.1007/s41669-021-00294-3.

Acknowledgements Biogen provided funding for medical writing support; Meryl Mandle from Excel Medical Affairs wrote the first draft of the manuscript based on input from the authors, and Miranda Dixon from Excel Medical Affairs copyedited and styled the manuscript per journal requirements.

\section{Declarations}

Funding This study was funded by Biogen.

Conflicts of interest/Competing interests Nicole Tsao was an employee of Biogen at the time of the study and owns stock in Biogen. Qiang Hou and Shih-Yin Chen are employees of and own stock in Biogen. Steven R. Messe is the local Principal Investigator for the Biogen CHARM trial to Evaluate the Efficacy and Safety of Intravenous BIIB093 (Glibenclamide) for Severe Cerebral Edema Following Large Hemispheric Infarction (NCT02864953).

Ethical approval This article does not contain any studies with human participants performed by any of the authors.

Consent to participate Not applicable.

Consent for publication Not applicable.

Availability of data and material Data used in this study are commercially available from IBM MarketScan.

Code availability Software application or custom code.
Author contributions NT and S-YC provided the study concept and design. Data acquisition and analysis were performed by NT, S-YC and $\mathrm{QH}$. All authors interpreted the results, and contributed to the development of the first draft, commented on previous versions, and approved the final manuscript.

Open Access This article is licensed under a Creative Commons Attribution-NonCommercial 4.0 International License, which permits any non-commercial use, sharing, adaptation, distribution and reproduction in any medium or format, as long as you give appropriate credit to the original author(s) and the source, provide a link to the Creative Commons licence, and indicate if changes were made. The images or other third party material in this article are included in the article's Creative Commons licence, unless indicated otherwise in a credit line to the material. If material is not included in the article's Creative Commons licence and your intended use is not permitted by statutory regulation or exceeds the permitted use, you will need to obtain permission directly from the copyright holder. To view a copy of this licence, visit http://creativecommons.org/licenses/by-nc/4.0/.

\section{References}

1. Hao Z, Chang X, Zhou H, Lin S, Liu M. A cohort study of decompressive craniectomy for malignant middle cerebral artery infarction: a real-world experience in clinical practice. Medicine (Baltimore). 2015;94(25):e1039.

2. Wijdicks EF, Sheth KN, Carter BS, Greer DM, Kasner SE, Kimberly WT, et al. American Heart Association Stroke Council. Recommendations for the management of cerebral and cerebellar infarction with swelling: a statement for healthcare professionals from the American Heart Association/American Stroke Association. Stroke. 2014;45(4):1222-38.

3. Torbey MT, Bösel J, Rhoney DH, Rincon F, Staykov D, Amar AP, et al. Evidence-based guidelines for the management of large hemispheric infarction: a statement for health care professionals from the Neurocritical Care Society and the German Society for Neuro-intensive Care and Emergency Medicine. Neurocrit Care. 2015;22(1):146-64.

4. Virani SS, Alonso A, Benjamin EJ, Bittencourt MS, Callaway CW, Carson AP, et al. Heart disease and stroke statistics-2020 update: a report from the American Heart Association. Circulation. 2020;141(9):e139-596.

5. Wartenberg KE. Malignant middle cerebral artery infarction. Curr Opin Crit Care. 2012;18(2):152-63.

6. Liebeskind DS, Jüttler E, Shapovalov Y, Yegin A, Landen J, Jauch EC. Cerebral edema associated with large hemispheric infarction. Stroke. 2019;50(9):2619-25.

7. Treadwell SD, Thanvi B. Malignant middle cerebral artery (MCA) infarction: pathophysiology, diagnosis and management. Postgrad Med J. 2010;86(1014):235-42.

8. Gerriets T, Stolz E, König S, Babacan S, Fiss I, Jauss M, et al. Sonographic monitoring of midline shift in space-occupying stroke: an early outcome predictor. Stroke. 2001;32(2):442-7.

9. Hacke W, Schwab S, Horn M, Spranger M, De Georgia M, von Kummer R. 'Malignant' middle cerebral artery territory infarction: clinical course and prognostic signs. Arch Neurol. 1996;53(4):309-15.

10. Kasner SE, Demchuk AM, Berrouschot J, Schmutzhard E, Harms $\mathrm{L}$, Verro $\mathrm{P}$, et al. Predictors of fatal brain edema in massive hemispheric ischemic stroke. Stroke. 2001;32(9):2117-23.

11. Hofmeijer J, van der Worp HB, Kappelle LJ, Eshuis S, Algra A, Greving JP, HAMLET Steering Committee. Cost-effectiveness of 
surgical decompression for space-occupying hemispheric infarction. Stroke. 2013;44(10):2923-5.

12. Chen SY, Thompson-Leduc P, Sawyer RN Jr, Fakih I, Cheung $\mathrm{HC}$, Macheca M, et al. Outcomes and resource use of patients with large hemispheric infarction and cerebral edema: analysis of real-world data. Curr Med Res Opin. 2021;37(5):781-8.

13. Hansen L. White Paper: IBM MarketScan research databases for life sciences researchers. IBM Watson Health, Somers, NY. 2018. https://www.ibm.com/downloads/cas/0NKLE57Y. Accessed 6 May 2021.

14. Torbey MT, Bosel J, Rhoney DH, Rincon F, Staykov D, Amar AP, et al. Evidence-based guidelines for the management of large hemispheric infarction: a statement for health care professionals from the Neurocritical Care Society and the German Society for Neuro-intensive Care and Emergency Medicine. Neurocrit Care. 2015;22(1):146-64.

15. Li YP, Hou MZ, Lu GY, Ciccone N, Wang XD, Dong L, et al. Neurologic functional outcomes of decompressive hemicraniectomy versus conventional treatment for malignant middle cerebral artery infarction: a systematic review and meta-analysis. World Neurosurg. 2017;99:709-25.e3.

16. Jüttler E, Unterberg A, Woitzik J, Bösel J, Amiri H, Sakowitz OW, et al. DESTINY II Investigators. Hemicraniectomy in older patients with extensive middle-cerebral-artery stroke. N Engl J Med. 2014;370(12):1091-100.

17. Das S, Mitchell P, Ross N, Whitfield PC. Decompressive hemicraniectomy in the treatment of malignant middle cerebral artery infarction: a meta-analysis. World Neurosurg. 2019;123:8-16.
18. Wu S, Yuan R, Wang Y, Wei C, Zhang S, Yang X, et al. Early prediction of malignant brain edema after ischemic stroke. Stroke. 2018;49(12):2918-27.

19. Berrouschot J, Sterker M, Bettin S, Köster J, Schneider D. Mortality of space-occupying ('malignant') middle cerebral artery infarction under conservative intensive care. Intensive Care Med. 1998;24(6):620-3.

20. Yu JW, Choi J-H, Kim D-H, Cha J-K, Huh J-T. Outcome following decompressive craniectomy for malignant middle cerebral artery infarction in patients older than 70 years old. J Cerebrovasc Endovasc Neurosurg. 2012;14(2):65-74.

21. Vahedi K, Hofmeijer J, Juettler E, Vicaut E, George B, Algra $\mathrm{A}$, et al. Early decompressive surgery in malignant infarction of the middle cerebral artery: a pooled analysis of three randomised controlled trials. Lancet Neurol. 2007;6(3):215-22.

22. Powers WJ, Rabinstein AA, Ackerson T, Adeoye OM, Bambakidis NC, Becker K, et al. 2018 guidelines for the early management of patients with acute ischemic stroke: a guideline for healthcare professionals from the American Heart Association/American Stroke Association. Stroke. 2018;49(3):e46-110.

23. Minnerup J, Wersching H, Ringelstein EB, Heindel W, Niederstadt T, Schilling M, et al. Prediction of malignant middle cerebral artery infarction using computed tomography-based intracranial volume reserve measurements. Stroke. 2011;42(12):3403-9. 